\title{
SENSEI: Direct-Detection Constraints on Sub-GeV Dark Matter from a Shallow Underground Run Using a Prototype Skipper CCD
}

\author{
Orr Abramoff, ${ }^{1}$ Liron Barak, ${ }^{1}$ Itay M. Bloch, ${ }^{1}$ Luke Chaplinsky, ${ }^{2,3}$ Michael Crisler, ${ }^{4}$ Dawa, ${ }^{2,3}$ \\ Alex Drlica-Wagner, ${ }^{4}$ Rouven Essig, ${ }^{2}$ Juan Estrada, ${ }^{4}$ Erez Etzion, ${ }^{1}$ Guillermo Fernandez, ${ }^{4}$ Daniel Gift, ${ }^{2,3}$ \\ Miguel Sofo-Haro, ${ }^{4,5}$ Joseph Taenzer, ${ }^{1}$ Javier Tiffenberg, ${ }^{4}$ Tomer Volansky, ${ }^{1,6}$ and Tien-Tien Yu ${ }^{7}$
}

(SENSEI Collaboration)

\author{
${ }^{1}$ Raymond and Beverly Sackler School of Physics and Astronomy, Tel-Aviv University, Tel-Aviv 69978, Israel \\ ${ }^{2}$ C.N. Yang Institute for Theoretical Physics, Stony Brook University, Stony Brook, New York 11794, USA \\ ${ }^{3}$ Department of Physics and Astronomy, Stony Brook University, Stony Brook University, Stony Brook, New York 11794, USA \\ ${ }^{4}$ Fermi National Accelerator Laboratory, P.O. Box 500, Batavia, Illinois 60510, USA \\ ${ }^{5}$ Centro Atómico Bariloche and Instituto Balseiro, Comisión Nacional de Energía Atómica (CNEA), \\ Universidad Nacional de Cuyo (UNCUYO), Argentina \\ ${ }^{6}$ School of Natural Sciences, The Institute for Advanced Study, Princeton, New Jersey 08540, USA \\ ${ }^{7}$ Department of Physics and Institute of Theoretical Science, University of Oregon, Eugene, Oregon 97403, USA
}

(Received 1 February 2019; published 24 April 2019)

\begin{abstract}
We present new direct-detection constraints on $\mathrm{eV}$-to-GeV dark matter interacting with electrons using a prototype detector of the Sub-Electron-Noise Skipper-CCD Experimental Instrument. The results are based on data taken in the MINOS cavern at the Fermi National Accelerator Laboratory. We focus on data obtained with two distinct readout strategies. For the first strategy, we read out the Skipper CCD continuously, accumulating an exposure of $0.177 \mathrm{~g}$ day. While we observe no events containing three or more electrons, we find a large one- and two-electron background event rate, which we attribute to spurious events induced by the amplifier in the Skipper-CCD readout stage. For the second strategy, we take five sets of data in which we switch off all amplifiers while exposing the Skipper CCD for $120 \mathrm{ks}$, and then read out the data through the best prototype amplifier. We find a one-electron event rate of $(3.51 \pm 0.10) \times 10^{-3}$ events/pixel/day, which is almost 2 orders of magnitude lower than the one-electron event rate observed in the continuous-readout data, and a two-electron event rate of $\left(3.18_{-0.55}^{+0.86}\right) \times 10^{-5}$ events/pixel/day. We again observe no events containing three or more electrons, for an exposure of $0.069 \mathrm{~g}$ day. We use these data to derive world-leading constraints on dark matterelectron scattering for masses between $500 \mathrm{keV}$ and $5 \mathrm{MeV}$, and on dark-photon dark matter being absorbed by electrons for a range of masses below $12.4 \mathrm{eV}$.
\end{abstract}

DOI: $10.1103 /$ PhysRevLett.122.161801

Introduction.-Direct-detection experiments play an important role in our quest to understand the nature of dark matter (DM). While weakly interacting massive particles, with masses in the $10 \mathrm{GeV}$ to $1 \mathrm{TeV}$ range, have been the main target of these experiments, the existence of well-motivated DM candidates with eV-to-GeV masses has prompted theoretical and experimental effort to also probe this lower mass range [1]. This is possible, e.g., by

Published by the American Physical Society under the terms of the Creative Commons Attribution 4.0 International license. Further distribution of this work must maintain attribution to the author(s) and the published article's title, journal citation, and DOI. Funded by SCOAP ${ }^{3}$. searching for DM interactions with electrons in various target materials [2].

The Sub-Electron-Noise Skipper-CCD Experimental Instrument (SENSEI) is the first dedicated direct-detection experiment to search for $\mathrm{DM}$ in the eV-to-GeV range. SENSEI uses silicon Skipper charge-coupled devices (Skipper CCDs) consisting of $\mathcal{O}\left(10^{6}\right)$ pixels. An electron in the valence band of the silicon can be promoted to the conduction band after it scatters off a DM particle with mass above $\sim 500 \mathrm{keV}$, or after it absorbs a DM particle with mass above the silicon band gap of $\sim 1.2 \mathrm{eV}$ [2-8]. The excited electron subsequently relaxes to the bottom of the conduction band, creating an additional electron-hole pair for each $3.8 \mathrm{eV}$ of excitation energy above the band gap [9]. The resulting electron-hole pairs [10] are moved pixel by pixel to one of the 
Skipper CCD corners containing the ultralow-noise readout stages that precisely measure their number [11]. Throughout this Letter, we refer to each contiguous grouping of pixels containing one or more electrons as an "event".

Here we present results from a prototype Skipper CCD first placed in the MINOS cavern in June 2017, located about $104 \mathrm{~m}$ [12] underground at the Fermi National Accelerator Laboratory (FNAL). We demonstrate how the Skipper CCD can be operated in different running modes, allowing us to identify a previously unknown source of background, which arises from soft photons emitted by an amplifier operated at the readout stage. We present the resulting DM constraints and discuss the impact of different running modes on the observed instrumental background of one- and two-electron events. Results from a surface run with this prototype were presented in Ref. [13].

SENSEI prototype detector.-The SENSEI prototype detector (protoSENSEI) consists of a single Skipper CCD placed in a light-tight copper housing, with an active area of $1.086 \times 1.872 \mathrm{~cm}^{2}$ and a total active mass (before masking) of $0.0947 \mathrm{~g}$. This prototype sensor has four amplifiers as part of the readout stage located in the four corners of the Skipper $\mathrm{CCD}$, each with a distinct design and noise performance. The four amplifiers allow the Skipper CCD to be divided into four equal-sized quadrants, each consisting of 624 rows of 362 pixels. Each pixel has an area of $15 \times 15 \mu \mathrm{m}^{2}$, a thickness of $200 \mu \mathrm{m}$, and a mass of $1.0476 \times 10^{-7} \mathrm{~g}$. While each amplifier usually reads one quadrant, it is also possible to have two quadrants read out through one amplifier. One amplifier design has high noise from charge-misclassification problems, and we discarded all data from it. The electronics consists of a modified Monsoon system as described in Ref. [11]. All data presented below were obtained by measuring each pixel 800 times.

Data collection strategies. - We took various sets of data with different exposure times and readout modes to understand detector backgrounds and constrain DM interactions. The largest dataset was collected by reading the Skipper CCD "continuously" and in parallel with four amplifiers, with each amplifier reading a single quadrant independently. The exposure time of each pixel is given by the Skipper-CCD readout time, which is about $4.4 \mathrm{k}$ seconds. We noticed that all quadrants have an increasing density of one- and two-electron events closer to the long edge of the Skipper CCD, where the amplifier is located, suggesting that the amplifier is producing excess events. When reading, the voltages on the amplifier are adjusted rapidly while sampling the charge packet in each pixel. These voltage variations increase the base current of the amplifier, which increases the probability of producing infrared photons [14]. These infrared photons can reach the active SkipperCCD region and contribute to spurious events with decreasing profile towards the center of the detector; see Fig. 1. Since all rows are exposed for the same time to the amplifier when reading continuously, we first sum across

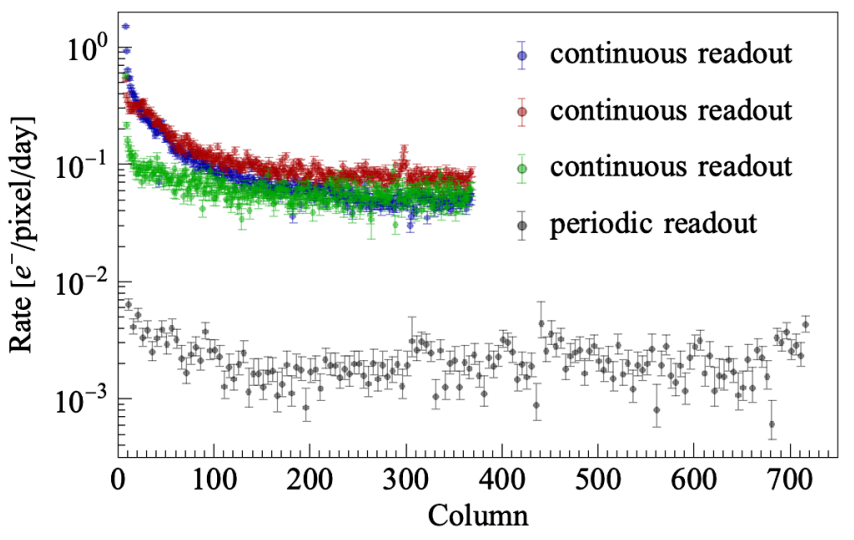

FIG. 1. The average measured one-electron rate as a function of the Skipper-CCD column number for the continuous-readout data (top three colored datasets, shown for the three working amplifiers) and for the periodic-readout data, in which two quadrants are read through the best amplifier (bottom black dataset; the latter was averaged and binned for five columns). Infrared photons produced by the amplifier (located near column 0) lead to an increasing background event rate in columns close to the amplifier. For the periodic readout, another amplifier is also located near column 724, but was turned off at all times.

all images in our dataset (discussed further below) the total number of one-electron events in a given column, and divide by the total number of pixels. Figure 1 shows the resulting mean number of one-electron events per pixel per day as a function of the column index for each of the three working amplifiers. We distinguish these "amplifierinduced events" from the "dark current," namely, fewelectron events that are distributed evenly across the Skipper-CCD region and are due to thermal fluctuations that occasionally promote valence-band electrons to the conduction band.

The observed rate of one-electron events depends on the distance from the amplifier, but is of order $\sim 0.1$ events/ pixel/day. We can still use the continuous-readout data to perform a background-free search for events with 3-100 electrons.

In order to reduce the excess of events from the amplifier, we took data by exposing the Skipper CCD for some time before reading it out periodically. During the exposure, the amplifiers were on but not actively moving or measuring charge packets, and we find that exposures of $30 \mathrm{k}-60 \mathrm{k}$ seconds reduce the one-electron rate by about an order of magnitude compared to the continuous-readout mode. We then took data for which the amplifiers were turned off during the exposure and only turned on to read out the data. We find another order of magnitude reduction in the oneelectron rate compared to the periodic-readout, amplifier-on data, suggesting that the amplifier is constantly emitting infrared photons, with the excess largest during readout. This is expected, since the base current is larger when the amplifier is actively reading. Finally, we took data with the amplifier off during the exposure for which we read out two 
quadrants through the amplifier with the best single-sample noise properties (yielding images with twice as many columns); see Fig. 1. While this did not reduce further the one-electron rate, we will present results below using five sets of data obtained in this readout mode, which we call periodic readout, each with an exposure of $120 \mathrm{k}$ seconds.

In summary, below we use a continuous-readout dataset to constrain the event rate of three and more electrons, and a periodic-readout dataset to constrain also the one- and twoelectron event rates. Since the detector is located at a shallow site, the image occupancy of the periodic-readout dataset, from multiple high-energy events, is high.

Data-quality cuts.-For both datasets, we use the same event-selection criteria as described in Ref. [13], together with additional quality cuts developed from our improved understanding of low-energy backgrounds and detector effects.

(i) Single-pixel events and neighbor mask. To simplify our analysis and reject multipixel events produced by random coincidence of one-electron pixels, we require the DM signal to be contained in a single pixel and only select pixels whose neighboring pixels are empty [13].

(ii) Electronic noise. We veto images in which the readout noise is 30\% larger than the expected readout noise as inferred from an overscan region in which virtual (nonexistent) pixels are read.

(iii) Edge mask. We remove eight pixels around the edge of each image to avoid any edge effects due to the nonuniformity in the electric field [15].

(iv) Bleeding zone mask. Because of charge-transfer inefficiency, we mask 50 pixels upstream in the vertical and horizontal direction of any pixel containing more than 100 electrons in the periodic-readout data. However, we observe that the charge-transfer inefficiency is slightly larger for charge shifts on the serial register (horizontal). To avoid any chance of spurious events with three or more electrons in the continuous-readout data (consisting of lowoccupancy images), we reject all pixels in the horizontal direction of pixels with more than 100 electrons (in addition to the 50 pixels in the vertical direction).

(v) Halo mask. The pixels around events with many electrons show an increased rate of low-energy events. We plan to study these in future work, but suspect that they are produced by infrared photons created by bremsstrahlung or recombination of ionized electrons. Here, we reject events located less than eight pixels away from any pixel containing more than 100 electrons.

(vi) Cross-talk mask. When reading the four quadrants simultaneously, high-energy signals recorded in one of the four quadrants can produce a fake signal in one or more of the other three due to electronic cross talk [16]. We reject pixels from all four quadrants that were read at the same time as a pixel containing more than 500 electrons.

(vii) Bad columns. Some pixels could contain defects or impurities that cause charge leakage, especially if a highenergy event occurs near such pixels. These appear as columns that contain an excess of nonempty pixels. To identify affected columns while keeping the analysis blind, we analyzed multiple sets of commissioning data exposing the Skipper CCD for 120k seconds each. Moreover, we analyzed the 50 masked pixels immediately above the highenergy events in the data used to derive our limits. Altogether we conservatively discard 60 "bad columns" whose noise was more than 2.5 standard deviations above the median noise across all columns averaged over all images.

All cuts above were developed on commissioning data or, in the case of the "bad column" cut, in the masked region of the physics data, and then applied to the unmasked physics data regions. An example of a masked image (but without the bad-column cut) is shown in Fig. 2. A summary of selection efficiencies is listed in Table I for electron bins $1-5$. Bins $1-3$ provide the best constraint on DM-electron scattering, while we use bins 1-100 to constrain DM absorption. The number of events with a given number of electrons is determined from a fit, since at only 800 samples per pixel in this prototype sensor there is some smearing from neighboring electron bins. We now discuss the analysis of each dataset in turn.

Continuous-readout data analysis.-These data consist of images from the three working amplifiers taken over 3.8 days, corresponding to $0.27 \mathrm{~g}$ day, which we use to constrain the rate of events containing 3-100 electrons.

Despite the excess events being produced by the amplifier, we can fiducialize the images by removing pixels that are too close to the amplifier and find the optimal constraint on the three-electron event rate. To do this, we must remove several columns close to the amplifier. We design the optimal column cut (after masking) using Fig. 1. We assume that the excess events produced by the amplifier follow a Poisson distribution, and predict the number of threeelectron events that would remain in the entire dataset as a function of the column index. We find that the minimum column indices for the three amplifiers that maximize the

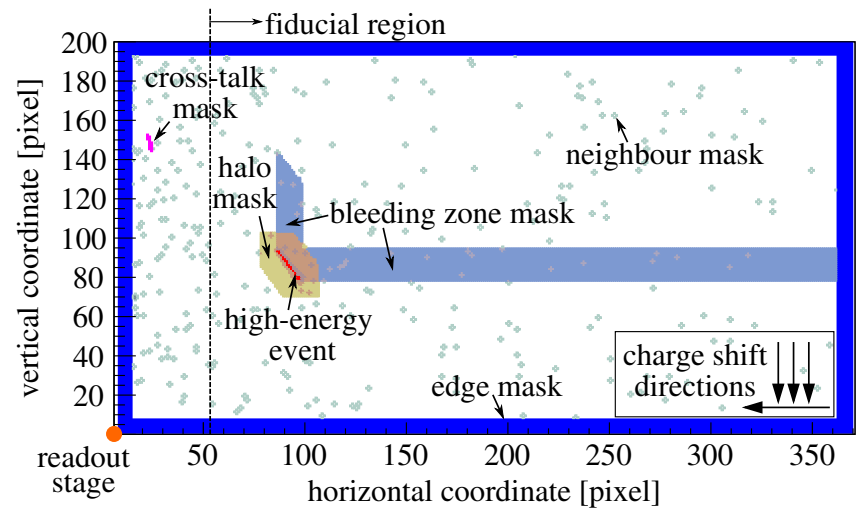

FIG. 2. Example of an image consisting of 200 rows from the continuous-readout data showing events and the mask after applying data-quality cuts. (The bad-column cut is not shown for presentation purposes only.) 
TABLE I. Efficiencies for the data-selection cuts for the periodic-readout and continuous-readout datasets, for events with 1-5 electrons. The bottom two rows list the efficiency-corrected exposures and the number of observed events after cuts, respectively.

\begin{tabular}{|c|c|c|c|c|c|c|}
\hline \multicolumn{7}{|c|}{$N_{e}$} \\
\hline \multirow[b]{2}{*}{ Cuts } & \multicolumn{3}{|c|}{ Periodic } & \multicolumn{3}{|c|}{ Continuous } \\
\hline & 1 & 2 & 3 & 3 & 4 & 5 \\
\hline $\begin{array}{l}\text { 1. DM in single } \\
\text { pixel }\end{array}$ & 1 & 0.62 & 0.48 & 0.48 & 0.41 & 0.36 \\
\hline 2. Nearest neighbor & & 0.92 & & & 0.96 & \\
\hline 3. Electronic noise & & 1 & & & $\sim 1$ & \\
\hline 4. Edge & & 0.92 & & & 0.88 & \\
\hline 5. Bleeding & & 0.71 & & & 0.98 & \\
\hline 6. Halo & & 0.80 & & & 0.99 & \\
\hline 7. Cross talk & & 0.99 & & & $\sim 1$ & \\
\hline 8. Bad columns & & 0.80 & & & 0.94 & \\
\hline Total efficiency & 0.38 & 0.24 & 0.18 & 0.37 & 0.31 & 0.28 \\
\hline Eff. expo. [g day] & 0.069 & 0.043 & 0.033 & 0.085 & 0.073 & 0.064 \\
\hline No. events & 2353 & 21 & 0 & 0 & 0 & 0 \\
\hline
\end{tabular}

total exposure time and predict not more than 0.5 threeelectron events are 55, 10, and 53, respectively. After applying these column cuts, we unblind and find the spectra shown in Fig. 3 (left). We find zero events with three (or more) electrons in the unblinded data. The final exposures after all data cuts (in g day) for each quadrant are 0.058, 0.067 , and 0.052 , respectively, for a total of $0.177 \mathrm{~g}$ day.

Periodic-readout data analysis.-We took five sets of $120 \mathrm{k}$-second-exposure, double-quadrant-readout data. After applying the data-quality cuts, each dataset is divided into three images of 200 rows each. To constrain the one-electron event rate, we apply additional data-selection criteria, which were determined from analyzing other $120 \mathrm{k}$-second-exposure commissioning data. First, we remove all five images that were read out last, since these have the longest exposure to the amplifier during readout. We then calculate the rate of events containing five or fewer electrons inside the masked regions of the remaining ten images, which we found in commissioning data to be positively correlated with the one-electron event rate outside the masked regions. We took the four images with the lowest rate in the masked region (since choosing four images gave the best constraint in the commissioning data), and then measured their average one-electron event rate outside the masked regions, finding $(3.51 \pm 0.10) \times 10^{-3}$ events $/ \mathrm{pixel} /$ day, with a $90 \%$ C.L. upper limit of $3.68 \times 10^{-3}$ events/pixel/day.

To constrain the two-electron event rate, we take the observed number of one-electron events in each of the ten images closest to the amplifier, and calculate the expected number of two-electron events in each of these images, assuming a Poisson distribution (the actual two-electron event rate in the data remained blinded during this procedure). We find that including the eight images with the lowest one-electron rate yields the lowest expected 90\% C.L. limit on the two-electron event rate, and an expected $\sim 6.5$ two-electron events. After unblinding these eight images, we find 21 events and a two-electron event rate of $\left(3.18_{-0.55}^{+0.86}\right) \times$ $10^{-5}$ events/pixel/day. This is more than expected, which we find is attributable to an insufficient masking of these high-occupancy images. Nevertheless, we include all observed two-electron events to find a $90 \%$ C.L. upper limit of $4.27 \times 10^{-5}$ two-electron events/pixel/day. The measured exposure (after all cuts) is $0.069 \mathrm{~g}$ day. The observed spectrum of events from these eight images is shown in Fig. 3 (right). We see no events with 3-100 electrons, and add this periodic-readout data to the continuousreadout data to constrain DM that produces 3-100 electrons, for a combined exposure of $0.246 \mathrm{~g}$ day.

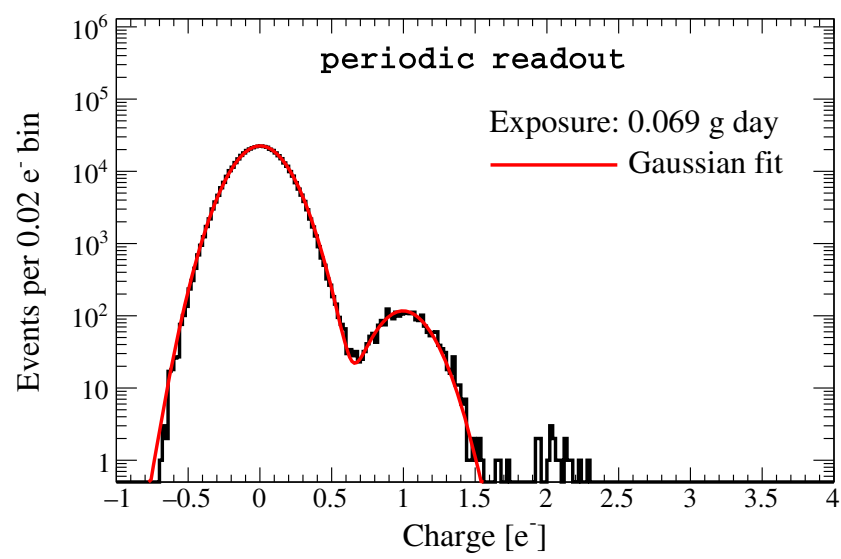

FIG. 3. Spectra of the recorded events for the continuous-readout (left) and periodic-readout (right) data. For the continuous-readout data, we show the spectra recorded by the three working amplifiers. The widths of the charge distributions depend on the amplifier design. The periodic-readout spectrum corresponds to the total number of events found in the eight double-quadrant images used to constrain the rate of events containing two and more electrons. There are no events with measured charge greater than 2.5 electrons in either data. Exposures include all efficiencies except for "cut 1" from Table I. 

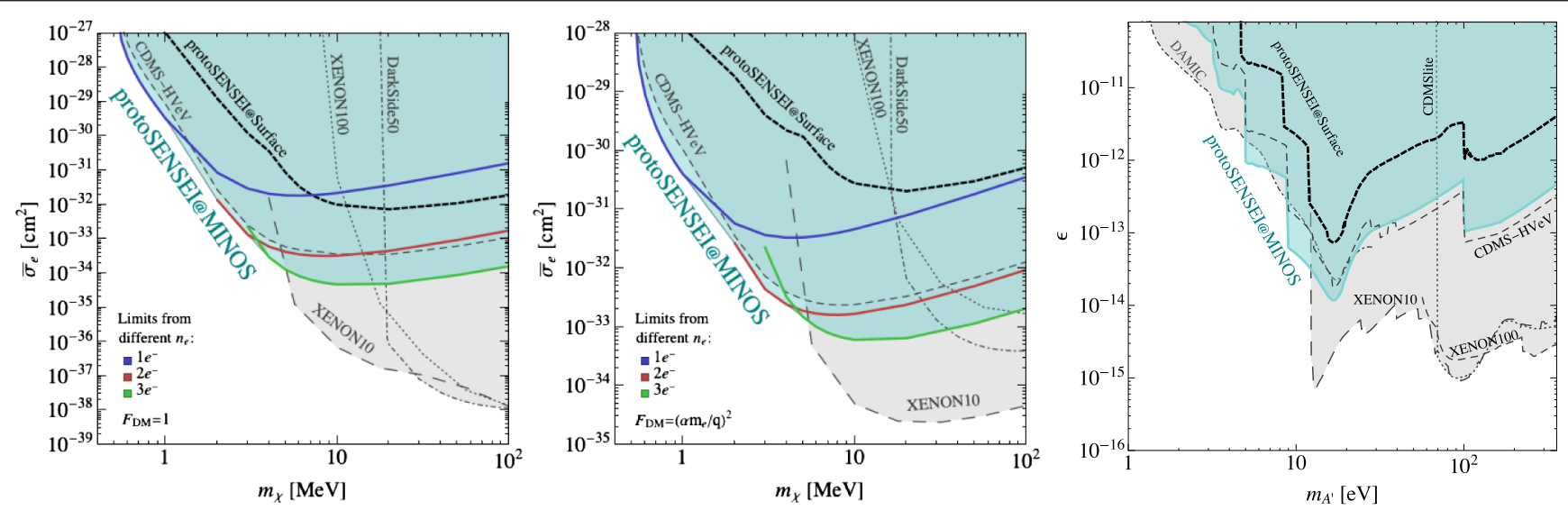

FIG. 4. The $90 \%$ C.L. constraints (cyan shaded regions) from a SENSEI prototype detector located underground near the MINOS cavern at FNAL. We show constraints on the DM-electron scattering cross section $\bar{\sigma}_{e}$ as a function of DM mass $m_{\chi}$ for two different DM form factors, $F_{\mathrm{DM}}(q)=1$ (left) and $F_{\mathrm{DM}}(q)=\left(\alpha m_{e} / q\right)^{2}$ (middle), and constraints on the kinetic-mixing parameter $\epsilon$ versus the darkphoton mass $m_{A^{\prime}}$ for dark-photon-dark-matter absorption (right). The thick blue and red lines use the one- and two-electron-rate constraints from the periodic-readout data, respectively, while the green line combines the three-electron-rate constraints from the continuous-readout and periodic-readout data. Constraints are also shown from the SENSEI surface run [13], XENON10 and XENON100 [22], DarkSide-50 [23], and CDMS-HVeV (without Fano-factor fluctuations, which is the upper bound of the band shown in Ref. [24]) for the left and middle plots, and from the SENSEI surface run [13], DAMIC [25], XENON10, XENON100, and CDMSlite [7] for the absorption limits on the right plot.

Dark matter results.-In Fig. 4, we show 90\% C.L. upper limits on the DM-electron scattering cross section $[2,3]$ and dark-photon dark matter absorption [6-8] using photoabsorption data from Refs. [17-19]. We assume a local DM density of $\rho_{\mathrm{DM}}=0.3 \mathrm{GeV} / \mathrm{cm}^{3}$ [20], a standard isothermal Maxwellian velocity distribution [21] with a DM escape velocity of $544 \mathrm{~km} / \mathrm{s}$, and a mean local velocity of $220 \mathrm{~km} / \mathrm{s}$. To be conservative, we do not include Fanofactor fluctuations.

For DM-electron scattering, $m_{\chi} \lesssim 1 \mathrm{MeV}\left(1 \lesssim m_{\chi} \lesssim\right.$ $4 \mathrm{MeV}$ ) is constrained most stringently by the observed one-electron (two-electron) event rate in the periodicreadout data, while the combined continuous-readout and periodic-readout data provide the best SENSEI constraint for $m_{\chi}>0.4 \mathrm{MeV}$ from observing no three-electron events. These results provide the most stringent direct-detection constraints on DM-electron scattering for $500 \mathrm{keV} \lesssim$ $m_{\chi} \lesssim 5 \mathrm{MeV}$. For DM absorption, SENSEI now provides the strongest constraint for some range of masses below $12.4 \mathrm{eV}$.

Outlook.-The SENSEI Collaboration is procuring $\sim 100 \mathrm{~g}$ of new Skipper CCDs and custom-designing electronics for an experiment at SNOLAB (Sudbury, Canada) that will be installed later this year. We expect these new sensors to have an improved noise performance and lower dark-count rate due to the use of higher-quality silicon. We are implementing mitigation strategies for amplifier-induced events based on a combination of optimizing the exposure time, readout-stage voltages, and fiducialization, and exploiting the elongated form factor of new detectors.
We thank Belina von Krosigk and Matthew Wilson for useful discussions. We are grateful for the support of the Heising-Simons Foundation under Grant No. 79921. R. E. also acknowledges support from DOE Grant No. DESC0017938. This work was supported by Fermilab under DOE Contract No. DE-AC02-07CH11359. The work of T. V. and E. E. is supported by the I-CORE Program of the Planning Budgeting Committee and the Israel Science Foundation (Grant No. 1937/12). T. V. is further supported by the European Research Council (ERC) under the EU Horizon 2020 Programme (ERC-CoG-2015-Proposal No. 682676 LDMThExp), and a grant from The Ambrose Monell Foundation, given by the Institute for Advanced Study. This Letter has been authored by Fermi Research Alliance, LLC under Contract No. DE-AC02-07CH11359 with the U.S. Department of Energy, Office of Science, Office of High Energy Physics. The U.S. Government retains, and the publisher, by accepting the article for publication, acknowledges that the U.S. Government retains a nonexclusive, paid-up, irrevocable, worldwide license to publish or reproduce the published form of this manuscript, or allow others to do so, for U.S. Government purposes.

[1] M. Battaglieri et al., arXiv:1707.04591.

[2] R. Essig, J. Mardon, and T. Volansky, Phys. Rev. D 85, 076007 (2012).

[3] R. Essig, M. Fernandez-Serra, J. Mardon, A. Soto, T. Volansky, and T.-T. Yu, J. High Energy Phys. 05 (2016) 046.

[4] S. K. Lee, M. Lisanti, S. Mishra-Sharma, and B. R. Safdi, Phys. Rev. D 92, 083517 (2015). 
[5] P. W. Graham, D. E. Kaplan, S. Rajendran, and M. T. Walters, Phys. Dark Universe 1, 32 (2012).

[6] H. An, M. Pospelov, J. Pradler, and A. Ritz, Phys. Lett. B 747, 331 (2015).

[7] I. M. Bloch, R. Essig, K. Tobioka, T. Volansky, and T.-T. Yu, J. High Energy Phys. 06 (2017) 087.

[8] Y. Hochberg, T. Lin, and K. M. Zurek, Phys. Rev. D 95, 023013 (2017).

[9] V. S. Vavilov, Sov. Phys. Usp. 4, 761 (1962).

[10] Below, we refer to electron-hole pairs simply as electrons.

[11] J. Tiffenberg, M. Sofo-Haro, A. Drlica-Wagner, R. Essig, Y. Guardincerri, S. Holland, T. Volansky, and T.-T. Yu, Phys. Rev. Lett. 119, 131802 (2017).

[12] P. Adamson et al. (MINOS Collaboration), Phys. Rev. D 77, 072002 (2008).

[13] M. Crisler, R. Essig, J. Estrada, G. Fernandez, J. Tiffenberg, M. S. Haro, T. Volansky, and T.-T. Yu (SENSEI Collaboration), Phys. Rev. Lett. 121, 061803 (2018).

[14] A. Toriumi, M. Yoshimi, M. Iwase, Y. Akiyama, and K. Taniguchi, IEEE Trans. Electron Devices 34, 1501 (1987).
[15] A. A. Plazas, G. M. Bernstein, and E. S. Sheldon, J. Instrum. 9, C04001 (2014).

[16] G. M. Bernstein et al., Publ. Astron. Soc. Pac. 129, 114502 (2017).

[17] E. B. Saloman and J. H. Hubbel (1986), https://inis.iaea.org/ search/search.aspx?orig_q=RN:18048512.

[18] X-ray interactions with matter, http://henke.lbl.gov/ optical_constants/.

[19] D. F. Edwards, in Handbook of Optical Constants of Solids, edited by E. D. Palik (Academic Press, Boston, 1985), pp. 547-569.

[20] J. Bovy and S. Tremaine, Astrophys. J. 756, 89 (2012).

[21] J. D. Lewin and P. F. Smith, Astropart. Phys. 6, 87 (1996).

[22] R. Essig, T. Volansky, and T.-T. Yu, Phys. Rev. D 96, 043017 (2017).

[23] P. Agnes et al. (DarkSide Collaboration), Phys. Rev. Lett. 121, 111303 (2018).

[24] R. Agnese et al. (SuperCDMS Collaboration), Phys. Rev. Lett. 121, 051301 (2018).

[25] A. Aguilar-Arevalo et al. (DAMIC Collaboration), Phys. Rev. Lett. 118, 141803 (2017). 